\title{
Torn ACL: a new bioengineered substitute brought from the laboratory to the knee joint
}

\author{
Francine Goulet, ${ }^{1,2}$ Denis Rancourt, ${ }^{3}$ Réjean Cloutier, ${ }^{1,2}$ Pierrot Tremblay, ${ }^{1,2}$ Anne-Marie Belzil, ${ }^{1,2}$ \\ Jean Lamontagne, ${ }^{1,2}$ Marc Bouchard, ${ }^{1,2}$ Julie Tremblay, ${ }^{1,2}$ Louis-Mathieu Stevens, ${ }^{1,2}$ Julie Labrosse, ${ }^{4}$ \\ Eve Langelier, ${ }^{3}$ Marc D McKee ${ }^{4}$ \\ 'Laboratory of Tissue Engineering, Hôpital de l'Enfant-Jésus, Quebec, QC, Canada; ${ }^{2}$ Department of Rehabilitation, Laval \\ University, Quebec, QC, Canada; ${ }^{3}$ Department of Mechanical Engineering, Laval University, Quebec, QC, Canada; \\ ${ }^{4}$ Faculty of Dentistry, and Department of Anatomy and Cell Biology, Faculty of Medicine, McGill University, Montreal, \\ QC, Canada
}

\begin{abstract}
Anterior cruciate ligament (ACL) injuries occur at an annual rate of 120000 in the USA, and many need reconstructive surgery. We report successful results at 1-13 months following implantation of bioengineered ACL (bACL) in goats. A bACL has been developed using autologous ACL cells, a collagen matrix and bone plugs. The extremities of the bACL were fully integrated into the femur and tibia of the host. Vascularisation of the grafts was extensive 1 month post-surgery and improved with time. At 6 months postgrafting, histological and ultrastructural observations demonstrated a highly organised ligamentous structure, rich in type I collagen fibres and fibroblasts. At the implants' insertion sites, characteristic fibrocartilage was observed having well aligned chondrocytes and collagen fibrils. After a year, mechanical rupture of the grafts demonstrated a major gain in strength. Eventual applications of this new technology in humans include multiple uses in orthopaedic, dental and reconstructive surgeries.
\end{abstract}

Keywords: ligament substitute, tissue engineering, knee joint, connective tissue, collagen

\section{Introduction}

In orthopaedics, knee trauma often involves the anterior cruciate ligament (ACL), and the reconstructive options are limited (Olson et al 1988; Sabiston et al 1990; Woods et al 1991; Jackson, Corsetti et al 1996; Jackson, Simon et al 1996; Frank and Jackson 1997; Hiemstra et al 2000). Novel bioengineering approaches create new adaptive alternatives for tissue replacement in several medical fields (Huynh et al 1999; Petite et al 2000). Tissue engineering offers the opportunity to target and control various biophysiological parameters of tissue development to make it competent for implantation; for example, collagen fibres and cells can be aligned in culture under defined conditions (Huang et al 1993; Kanda and Matsuda 1994; Black et al 1998; Paquette et al 1998; Kim et al 1999; Goulet et al 2000). Perhaps most importantly, bioengineered tissue substitutes are expected to respond and adapt to the mechanical stresses that occur following implantation in vivo.

Few attempts at creating a bioengineered ACL (bACL) have been reported (Dunn et al 1992, 1993, 1994). A drawback of Dunn's approach (Dunn et al 1994) is that the matrix of the graft cannot be quickly degraded, and its remodelling is impaired. Bellincampi et al (1998) added autologous cells to such scaffolds, but they were nearly resorbed by 8 weeks post-implantation. This example suggests that the stability of the collagen scaffold is a critical parameter to monitor in vivo. The best option for permanent human ACL replacement is not yet defined (Frank and Jackson 1997; Fu et al 1999).

To our knowledge, permanent and successful implantation of bioengineered ligaments grown from a culture dish has not yet been reported. The bACL used in this research is unique, as it is made of collagen in which living cells are seeded, not around the matrix but within it (Goulet, Germain et al 1997; Goulet, Rancourt et al 1997; Goulet et al 2000). This paper describes successful results obtained after long-term bACL implantation in goats.

Correspondence: Francine Goulet, Laboratoire de Génie Tissulaire, Pavillon Notre-Dame, H-40 I, I40 I $18^{\mathrm{e}}$ rue, Quebec, QC GIJ IZ4, Canada; tel + | $4 \mid 86490252$ ext 4344; fax + | 4|8649 5969; email chgfgo@hermes.ulaval.ca 


\section{Experimental protocol Isolation and culture of GLFs}

To isolate autologous goat ligament fibroblasts (GLFs), female adult goat knee ACLs were totally resected from their osseous insertion sites. The two insertion sites on the femur and tibia were carefully scraped down to the mineralised tissue with a scalpel to ensure that no residual pieces of ACL remained in situ. These sites were also marked for the future insertion of the autologous bACL. The ACL was temporarily maintained at $4{ }^{\circ} \mathrm{C}$ in serum-free Dulbecco's modification of Eagle's medium (DMEM) (Gibco BRL, Life Technologies, Grand Island, NY, USA), and cells were isolated and cultured as previously described (Goulet, Germain et al 1997; Goulet, Rancourt et al 1997; Goulet et al 2000). Briefly, goat ACL biopsies were weighted and cut into small pieces after removal of the periligamentous tissue. The fragments were digested with $0.125 \%$ collagenase, containing $2 \mathrm{mmol} / \mathrm{L} \mathrm{CaCl}_{2}(1 \mathrm{~mL}$ of enzymatic solution per milligram of tissue) for $20 \mathrm{~h}$, under gentle agitation at $37^{\circ} \mathrm{C}$. A $0.1 \%$ trypsin solution $(1 \mathrm{~mL}$ per milligram of hydrated tissue) was then added to the cellular suspension for $1 \mathrm{~h}$. The enzymes were dissolved in DMEM, pH 7.4, containing antibiotics. The GLFs were cultured in DMEM supplemented with $10 \%$ foetal calf serum (FCS) and antibiotics. When GLF primary cultures reached $85 \%$ confluence, the cells were detached from their culture flasks using $0.05 \%$ trypsin, $0.01 \%$ EDTA solution $(\mathrm{pH} 7.8$ ) for about $10 \mathrm{~min}$ at $37^{\circ} \mathrm{C}$. GLF suspensions were centrifuged twice at $200 \mathrm{~g}$ for $10 \mathrm{~min}$. The cell pellets were resuspended in culture medium, and the GLFs were counted with a Coulter Counter $^{\circledR}$ and Multisizer ${ }^{\mathrm{TM}}$ analyser. The cellular viability was determined using the trypan blue exclusion method (always $>90 \%$ viable cells). The GLFs maintained their morphology for at least 7 passages in culture and secreted types I and III collagens and glycosaminoglycans in monolayers (data not shown). All procedures were approved by the local ethics committee.

\section{Preparation of autologous graftable $b A C L$}

To achieve the permanent fixation of the ACL to the bones, cylindrically shaped porcine bone plugs were prepared $(1 \mathrm{~cm}$ diameter by $2 \mathrm{~cm}$ long) and pierced with a transverse hole (3.2 $\mathrm{mm}$ diameter). They were rinsed and stored in 100\% ethanol for 2-3 days to eliminate traces of blood and to achieve sterilisation. A polyglyconate MAXON surgical thread (size 3-0; Sherwood-Davis \& Geck, St Louis, MO, USA), resorbable within 4-6 weeks post-surgery, was passed through the holes in the two bone plugs and tied. The bones and thread were counter-rotated to provide a single twisted-thread link between the plugs. This bone/ thread scaffolding was transferred to a sterile plastic tube and kept extended in a central, suspended position by passing two metal pins across the tube and through the transverse holes in the bone plugs.

For casting the bACLs, DMEM containing FCS and $1.0 \mathrm{mg} / \mathrm{mL}$ of bovine type I collagen (isolated in our laboratory from healthy Canadian beef skin, tested for its purity by electrophoresis, and solubilised in acetic acid diluted 1000 times with sterile water) was quickly mixed with a suspension of autologous GLFs $\left(2.5 \times 10^{5} \mathrm{cells} / \mathrm{mL}\right)$. The mixture (total of $10 \mathrm{~mL}$ ) was poured into $12-\mathrm{mL}$ sterile plastic tubes containing the bone plugs linked by the surgical thread. The collagen polymerised in the mixture within $20 \mathrm{~min}$ at room temperature under a sterile culture flow hood and was maintained without any agitation. These bACLs were cultured for $24 \mathrm{~h}$ in DMEM supplemented with $10 \%$ FCS, $50 \mu \mathrm{g} / \mathrm{mL}$ ascorbic acid and antibiotics, during which time the collagen was contracted by the cells onto and around the twisted surgical thread. Each bACL was produced at a length of $25 \mathrm{~mm}$ in culture.

The bACLs were frozen in sterile petri dishes overnight at $-70^{\circ} \mathrm{C}$ and subsequently lyophilised. They were transferred back into new sterile plastic tubes and fixed again with pins as previously described. Following rehydration in fresh DMEM to produce a semi-rigid central core, a second coating of collagen and GLFs in solution was applied as described above. A bilayered bACL was obtained with a lyophilised core and a living cell-populated outer layer. The bACLs were viable prior to implantation, since the GLFs progressively contracted the outer collagen layer in vitro over $24 \mathrm{~h}$ and thereafter. The resultant bACLs were kept in culture for 6-8 days until grafted into their respective hosts.

\section{Surgical procedures for implantation of autologous $b A C L s$ into goats}

All surgical implantation procedures were performed under general anaesthesia on 45-kg goats whose native ACLs had been resected one month earlier. Upon surgical re-entry, knees appeared totally healed with no signs of overt inflammation, and all knee joints showed a complete range of motion.

With use of Kirschner wires and a mini-driver (Smith \& Nephew, QC, Canada), an angled tunnel having the same diameter as the bone plugs was drilled through the lateral 
side of the femur. The bACL was threaded through the femoral bone tunnel. The lead bone plug was then inserted into a second tunnel drilled into the tibia (Kurosaka et al 1987; Olson et al 1988). Both bone plugs were fixed in place with screws, and all incision sites were sprayed with a topical antibacterial agent prior to wound closure.

An immobilising plaster cast was placed on the leg during the first week post-surgery to limit motion (to favour healing) and to prevent the goat from irritating the wound. Following removal of the cast, the goats gradually returned to putting weight on the affected leg, walking freely thereafter. The animals were monitored daily by the veterinary team at the animal care unit.

A total of 12 bACLs were grafted into 10 goats. The first goat was grafted for 1 month; 4 goats, 6 months; and the 5 others, 11-13 months, with 2 goats receiving grafts in both knees (with an interval of 2 months). The implanted bACLs were removed under general anaesthesia.

\section{Histologic analysis of bACL after implantation}

Histologic studies were performed on bACLs before implantation; on whole bACLs, 1 and 6 months postimplantation; and on small peripheral punch biopsies from the 1-year end points. The bACL samples were fixed in an aldehyde-containing solution, embedded in either paraffin or LR White acrylic resin, and sectioned and stained by either Masson's trichrome method or toluidine blue. Histological sections stained by Masson's trichrome method were examined to assess averaged cell density in the bACLs. Micrographs of the sections, taken under phase contrast microscope, were transferred to a Dell computer. Morphometric analysis of each section was performed using the MetaMorph ${ }^{\circledR}$ imaging system (Universal Imaging Corporation $^{\mathrm{TM}}$, Downingtown, PA, USA) by counting the cells on different view fields $(n=4-5)$ on 3 different biopsies taken at the mid-length of bACLs, grafted for 1 and 6 months. Selected LR White samples were further sectioned for ultrastructural analysis by transmission electron microscopy (Nanci et al 1996). Briefly, biopsies were fixed for $1 \mathrm{~h}$ in a cacodylate-buffered $1.2 \%$ glutaraldehyde containing $0.5 \%$ ruthenium tetroxide and then post-fixed for $3 \mathrm{~h}$ in $5 \%$ osmium tetroxide, $5 \%$ ruthenium tetroxide in a cacodylate buffer. After dehydration, the samples were embedded in Epon 812. Contrasted sections were observed under a JEOL 1200EX transmission microscope (Peabody, MA, USA). All histological and ultrastructural analyses were performed on at least 3 different fields, on 3 tissue biopsies (blocks).

\section{Biomechanical analysis of bACLs after implantation}

A traction apparatus was designed to assess the mechanical properties of the bACL in culture (Langelier et al 1999). A commercial, materials-testing machine (Instron) was used to measure the ultimate strength of the grafts ex vivo and at post-mortem, after removing all the surrounding tendons and other anatomical structures (Figure 1a). All rupture tests (control and bACL groups) were performed at a fixed angle of $90^{\circ}$ between the tibia and the femur (Figure 1b). Rupturing was done at a constant rate displacement ramp $(1 \mathrm{~cm} / \mathrm{s})$ while being simultaneously recorded with a digital video camera. Some other rupture tests were subsequently performed to assess the strength of goat ACLs (healthy controls) by another approach, which involves the application of the tension in the direction of its long axis during the test.

\section{Statistical analyses}

The statistical comparison of the data was performed according to the student's t-test, using native ACLs as controls.

\section{Results and discussion}

GLFs isolated from autologous native ACL were grown and seeded in a bovine collagen matrix casted between 2 bone plugs linked by a resorbable surgical thread. Each bACL was lyophilised the following day to condense and reinforce the insertion of its collagen matrix into the bone plugs (Figure 2a). Following lyophilisation the GLFs died. The second collagen layer populated with autologous living GLFs, encompassing and attaching to the rehydrated lyophilised central core, formed a functionally stable bACL that was desirable for implantation (Figure 2b).

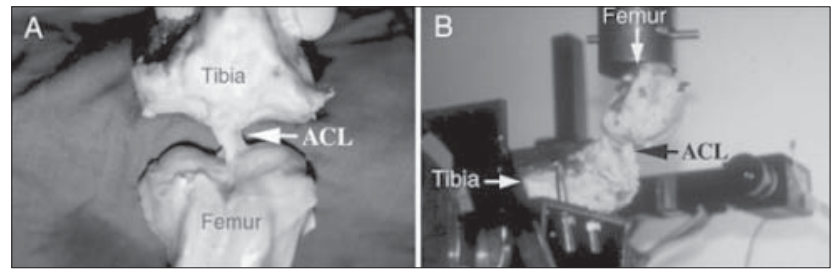

Figure I Macroscopic view of an II-month-old graft ex vivo as prepared for biomechanical testing before (a) and during (b) application of tension prior to rupture. 

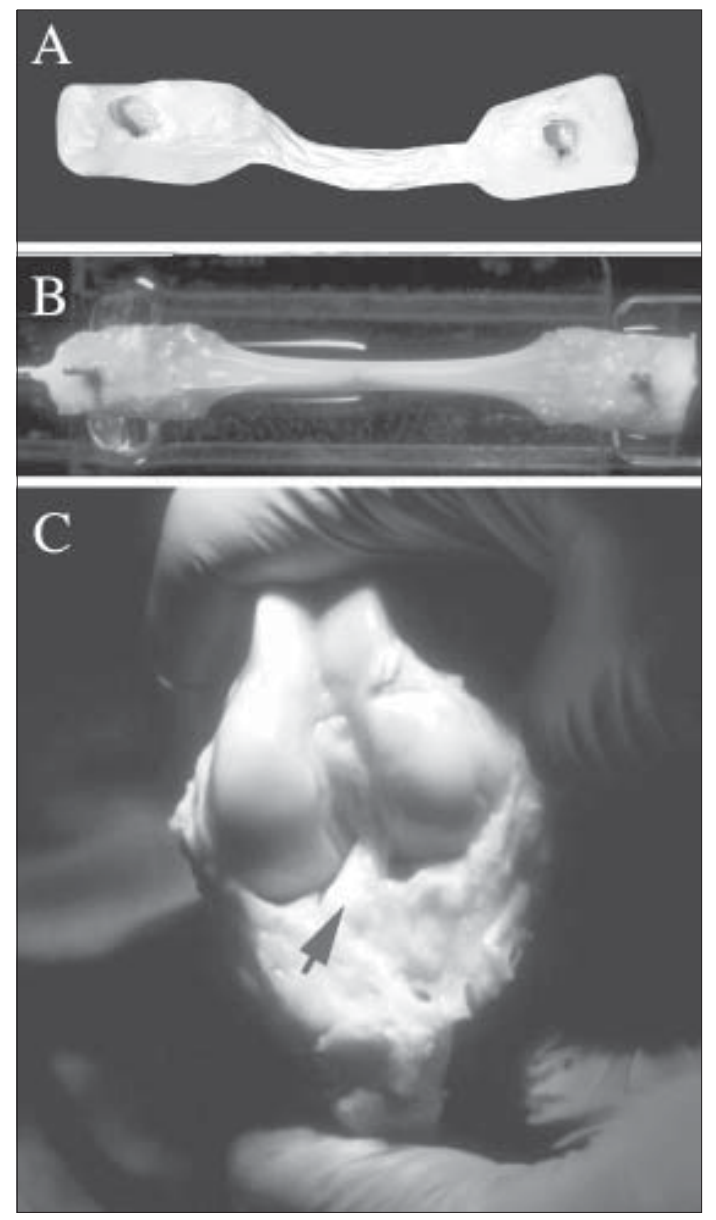

Figure 2 Construction of bACL and post-grafting view in situ. (a) Macroscopic appearance of the initial collagen layer contracted onto two bone plugs and lyophilised. (b) A bACL after the second collagen coating and ready for grafting. (c) Macroscopic view of a bACL in situ (arrow) grafted for one year into a goat knee.

It is the opinion of the authors that the gold standard for any tissue-engineered ACL is a native or unoperated goat's ACL. Consequently, a cultured bACL prior to implantation is rather considered as the most appropriate 'negative control'. In the initial experiment, at one month post-grafting of the autologous bACL, the goat was highly active with no signs of inflammation, and there was full weight-bearing and even jumping using the affected leg. On the basis of these observations, autologous bACLs were grafted for longer intervals up to a year. In all cases, post-mortem macroscopic inspection of the grafted knee joints revealed implants with highly comparable anatomical proportions and relationships to the contralateral native goat ACLs (Figure 2c). The bACLs remained oriented as grafted, without being overstretched or loose in the knee joints. No signs of articular cartilage degeneration were observed in any of the grafted knees, and histological analyses confirmed the macroscopic observations. In situ, the bACL went through a remodelling process, 'ligamentisation', similar to what has been observed in autografts using the central portion of the patellar tendon (Amiel et al 1986; Sabiston et al 1990; Frank and Jackson 1997; Hiemstra et al 2000). Ligamentisation occurs after the vascularisation of the graft and involves gradual assumption of the microscopic properties of normal ACL, an increase in collagen concentration, the formation of bundles in the grafts and the regeneration of the fibrocartilage ligament insertions in the bones of the knee joint.

Light microscopic histological analysis revealed that at all levels of the ligament substance, dense and well organised collagen fibres and fibroblasts were readily apparent (Figure $3 a$ ), as in native ACL (Figure 3b). These observations suggest that the integrated GLFs in this living bACL play an important role in matrix remodelling in vitro, prior to and post-implantation.
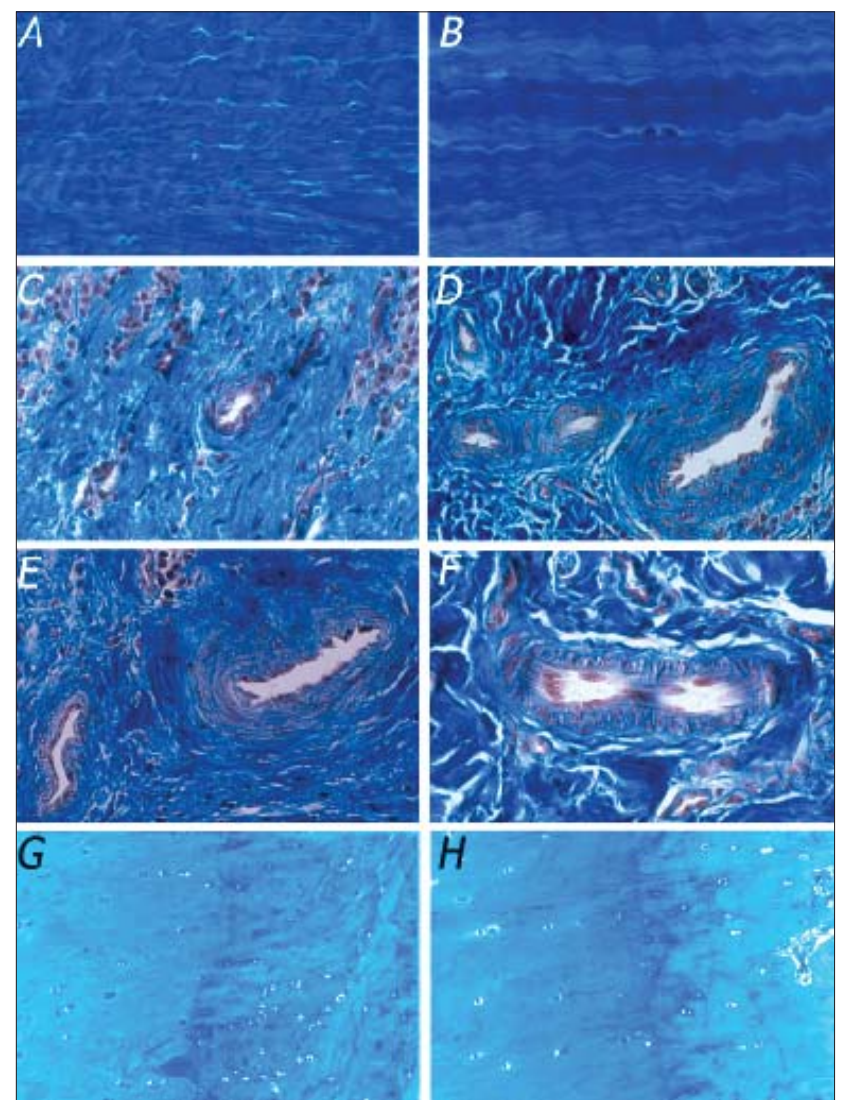

H

Figure 3 Light microscope histology of paraffin sections of bACL grafted in goat knee and stained with Masson's trichrome method showed a dense network of collagen fibres organised in a typical crimp pattern in bACL grafted for 13 months (a) and native ACL (b) (120 x magnification). Vascularisation observed in synovial membranes attached to bACLs grafted for I month (c), 6 months (d) and II months (e), compared with blood vessel in contralateral native ACL (f). Light microscope histology of tissue sections embedded in LR White and stained with toluidine blue showed fibrocartilage and chondrocytes at the junction of the ligament proper and the bone in both the bACL grafted for 6 months $(\mathrm{g})$ and native ACLs (h) (I50 × magnification). 
One month post-grafting, excellent integration of the implant into the goat knee and extensive vascularisation were observed (Figure 3c). Histological assessment of the bACLs after 6-12 months were all similar (Figures $3 \mathrm{~d}$ and $3 e$ ) when compared with native ACLs (Figure 3f). There were no signs of any suture as early as 5 weeks post-grafting. At six months post-grafting (Figure $3 \mathrm{~g}$ ), and like the contralateral control ACL (Figure 3h), chondrocytes were embedded in neosynthesised fibrocartilage located at the interface between the ligament proper and the bone plugs, with both experimental and control sides having collagen fibres aligned parallel to the long axis of the graft. It may be postulated that transplanted GLFs were differentiated into chondrocytes in situ, as it can occur in vitro under specific conditions (Yabu et al 1992; Hoshi et al 1997).

No histological differences were observed between the samples taken at the surface and within the deep layer of the bACLs in terms of cells and collagen fibre density and alignment. These data suggest that the matrix network was regenerated through the secretion of de novo synthesised collagen fibres by living ACL cells, well distributed in the ligament substance. The new collagen fibres could be

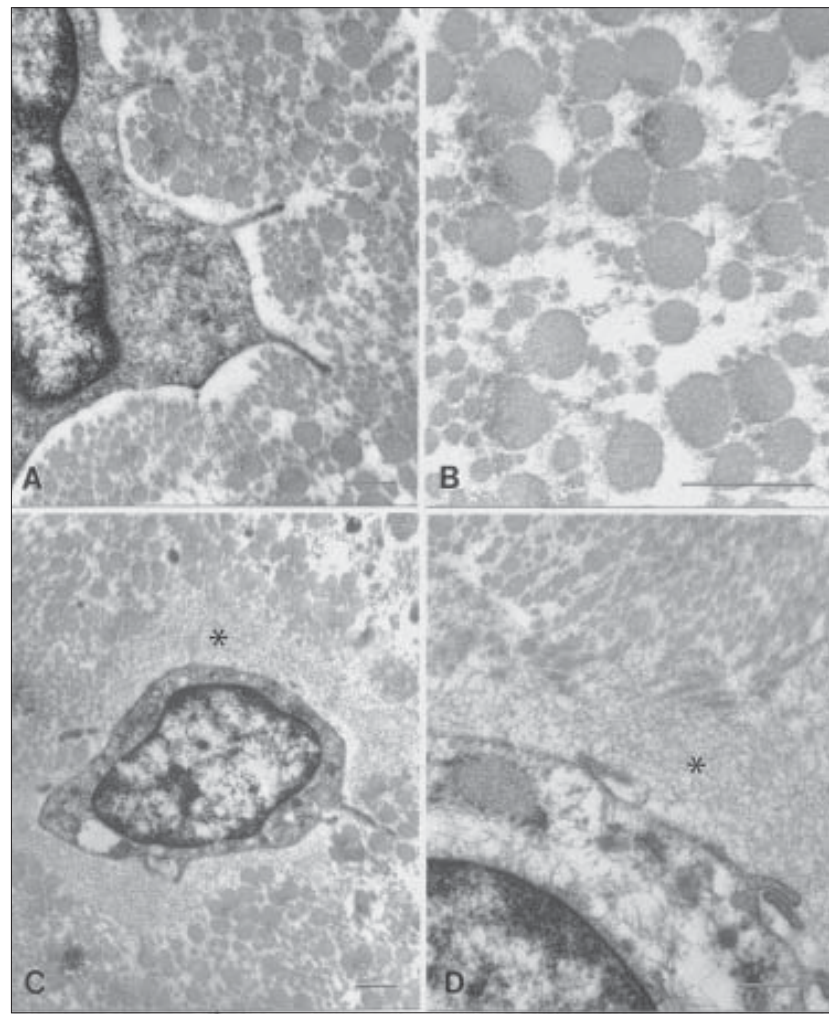

Figure 4 Transmission electron micrographs of a cross-sectioned bACL 6 months after grafting. Fibroblasts with short cytoplasmic extensions, which insert into an extracellular matrix having numerous collagen fibrils $(a, b)$. At the osseous insertion sites, bACL cells appear as chondrocytes surrounded by a network of proteoglycan aggregates $(*)(c, d)$. Bars equal $0.5 \mu \mathrm{m}$. produced by the cells seeded in the bACLs before grafting and/or by fibroblasts that migrated into the bACL scaffold from surrounding tissues (such as synoviocytes). The fate of GLFs and the possible recruitment of additional cells into the bACL scaffolds after various implantation periods is under investigation. However, cell density decreased with the regeneration of the bACLs. Similar observations were reported using allografts for ACL replacement (Jackson, Corsetti et al 1996). An average cell density of 879 cells/ $\mathrm{mm}^{2}(\mathrm{p}<0.1, \mathrm{n}=15) 1$ month post-grafting diminished to an average of 694 cells $/ \mathrm{mm}^{2}(\mathrm{p}<0.05, \mathrm{n}=12) 5$ months later Cell density at the mid-length of native ACLs, used as controls, corresponded to an average of 361 cells $/ \mathrm{mm}^{2}$ $(\mathrm{p}<0.05, \mathrm{n}=10)$. These data suggest that the bACLs underwent progressive regeneration to become eventually competent ACLs.

At 6 months post-implantation, and also at the 1-year end points, ultrastructural characteristics of the grafts (Figure 4) were comparable to contralateral native ACL (Figure 5). In the central portions of the bACL, fibroblasts were dispersed throughout a type I collagen-rich extracellular matrix. The cells showed cytoplasmic extensions

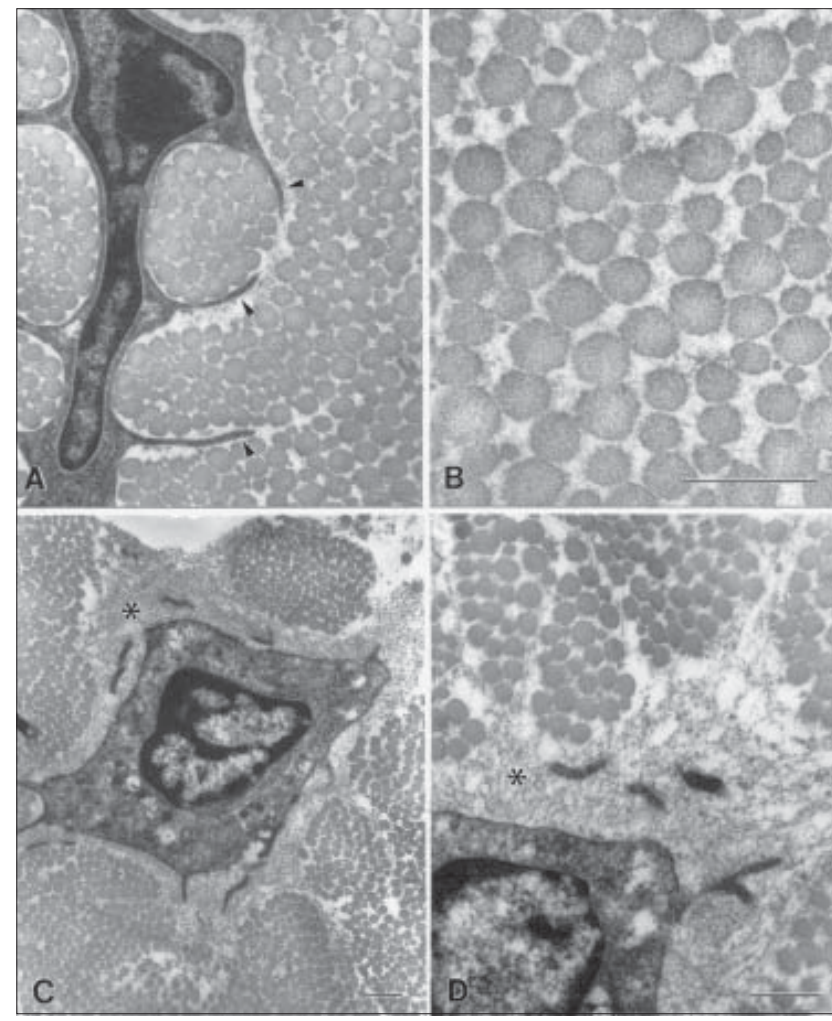

Figure 5 Transmission electron micrograph of a cross-sectioned, contralateral native ACL. Fibroblasts with short cytoplasmic extensions, which insert into an extracellular matrix having numerous collagen fibrils $(a, b)$. At the osseous insertion sites, bACL cells appear as chondrocytes surrounded by a network of proteoglycan aggregates $(*)(c, d)$. Bars equal $0.5 \mu \mathrm{m}$ 
typical of ligament cells inserting into the matrix (Figure $4 a)$, and collagen fibril diameters demonstrated some variability but many had large diameters typical of mature ligament (Figures $4 \mathrm{a}$ and $4 \mathrm{~b}$ ). The presence of large fibrils is very interesting, as most ligament grafts are repopulated with a unimodal distribution of small-diameter collagen fibrils, consistent with scar tissue. The presence of largediameter collagen fibrils suggests that the phenotype of the cells is more like normal ACL cells. In the contralateral control ACL, cells had more pronounced cytoplasmic extensions that compartmentalised collagen fibril groups (Figure 5a), and collagen fibril diameters were more homogeneously distributed, mostly of the larger size and more closely packed together than in the bACL (Figure 5b). At the insertion sites of both bACL (Figures $4 \mathrm{c}$ and $4 \mathrm{~d}$ ) and native ACL (Figures 5c and 5d), fibrocartilaginous tissue was apparent as evidenced by the pericellular accumulation of a fine, fibrillar network characteristic of proteoglycan aggregates. Immunohistochemical staining of these same regions were positive for the cartilage markers aggrecan and link protein (data not shown). Collectively, these data indicate that the bovine collagen matrix initially used to produce the bACL is progressively replaced by de novo synthesis of nascent extracellular matrix, without rejection by the host.

In terms of biomechanical strength, the retrieved grafts were surprisingly resistant to rupture under tension, a feature we believe attributable not only to long-term matrix remodelling but also to the large-size fibre formation. Manual testing of the knees' laxity performed before disarticulation, suggested that the grafted knees were as stable as the normal ones (data not shown). Other knee structures (eg tendons) contribute to the joint stabilisation, but the goats showed a good distribution of their body weight on both legs (operated and normal contralateral) when put on force platforms (data not shown).

In culture, in the absence of a lyophilised core and of the resorbable surgical thread, all bACL matrix would break at the bone-collagen interface at forces ranging from 0.2 to $0.5 \mathrm{~N}$. When a lyophilised central core was added, the bACL could support at most $2 \mathrm{~N}$ before rupture. The addition of a surgical thread to the lyophilised central core allowed the bACL to sustain up to $25 \mathrm{~N}$. However, this thread is degraded 4-6 weeks post-grafting. Therefore, the strength values measured 11-13 months post-implantation had to be compared with about $2 \mathrm{~N}$ (in absence of the thread). The average bACL strength from 7 grafted knees was $23 \%$ after
11 months and 36\% after 13 months, compared with the average ultimate strengths of native goat ACL corresponding to $505 \mathrm{~N}$ or $100 \%$. These data demonstrate that implanted bACLs gained considerable mechanical strength postimplantation when compared with contralateral healthy ACLs (they reached almost $40 \%$ of the native ACL strength). Interestingly, the highest rupture value was obtained for the bACL with the longest residence time in vivo (13 months). This is consistent with the hypothesis that bACLs continue to increase in strength with time post-grafting. The differences in collagen fibril density and diameter observed by electron microscopy at 6 months and 1 year post-grafting, together with possible differences in the extent of collagen cross-linking, likely explain the lower strength of the grafts relative to control ligaments. However, it is expected that the addition of a post-surgical joint-training regime might significantly reinforce such grafts, an otherwise obvious requirement in the event of human applications.

In summary, the data have shown the successful integration of our bACL at both bone extremities, its rapid vascularisation, the remodelling of the whole lyophilised matrix, the excellent collagen fibre alignment, the gain in number and diameter of matrix fibres and the acute neosynthesis of collagen-including fibrocartilage in situ. The 1-year-old grafts were compared with native ACL that have been in a goat's knee joint since birth. After only a year of implantation, we conclude that the data are promising. The number of samples tested in goats is low; these are heavy and expensive animals. Starting with a bACL that could not sustain more than $2 \mathrm{~N}$ in vitro, the gain in strength of all grafts can objectively be considered as $100 \%$ successful after 11-13 months.

The use of bACL presents several unique advantages compared with the more conventional alternatives typically used for torn ACL repair or replacement. First, unlike synthetic ligament prostheses, implantation of bACL is permanent. Second, our autologous technology avoids tissue morbidity, sparing the use of secondary surgical sites and tissues. Implantation of a bACL can be performed arthroscopically, avoiding arthrotomies and the associated risks of infection, major swelling and permanent tissue scarring. Such advantages could contribute to reduced medical costs on a long-term basis and improve the quality of life of thousands of patients in the near future. In the case of bACL failure, this method does not preclude the subsequent use of the more conventional reconstructive approaches. We do not pretend that we have found the only 
option for torn ACL replacement, but we sincerely believe that we have discovered a novel and promising approach that may provide solutions to this clinical problem.

\section{Acknowledgements}

We thank Dr Albert Normand, orthopaedic surgeon, who died early after the initiation of our work and was the first to propose the concept of a bioengineered ACL grown in vitro. We thank the Animal Care Unit of Laval University, notably Dr Jim Gourdon and Mario Mercier for precious assistance, Mr Flavio Marinelli from Smith \& Nephew for lending surgical instrumentation, Dr Nazrul Islam for his intellectual contribution during establishment of the method of collagen lyophilisation, Dr Aristidis Paterakis for computer programming, and Dominique Robitaille and Christine Plamondon for their practical contribution to the latest advances of this work. F Goulet is the recipient of a Scholarship from the FRSQ. This work was supported by the CIHR (grants \#14772 and \#49478 to F Goulet and D Rancourt), CFI (F Goulet), The Canadian Orthopaedic Association (R Cloutier and F Goulet), The RenaudLemieux Foundation of Saint-Sacrement Hospital and The Club Richelieu of Limoilou, Quebec, Canada.

\section{Notes}

The procedures leading to the production of similar products and/or applications were filed for patent in the USA $(09 / 990,320)$ and in Europe (PCT/CA01/01637).

\section{References}

Amiel D, Kleiner JB, Roux RD et al. 1986. The phenomenon of 'ligamentization': anterior cruciate ligament reconstruction with autogenous patellar tendon. J Orthop Res, 4:162-72.

Bellincampi LD, Closkey RF, Prasad R et al. 1998. Viability of fibroblastseeded ligament analogs after autogenous implantation. J Orthop Res, $16: 414-20$.

Black A, Berthod F, L'Heureux N et al. 1998. In vitro reconstruction of a human capillary-like network in a tissue-engineered skin equivalent. FASEB J, 12:133-40.

Dunn MG, Avasarala PN, Zawadsky JP. 1993. Optimization of extruded collagen fibers for ACL reconstruction. J Biomed Mater Res, 27: $1545-52$.

Dunn MG, Maxian SH, Zawadsky JP. 1994. Intraosseous incorporation of composite collagen prostheses designed for ligament reconstruction. J Orthop Res, 12:128-37.

Dunn MG, Tria AJ, Kato YP et al. 1992. Anterior cruciate ligament reconstruction using a composite collagenous prosthesis: a biomechanical and histologic study in rabbits. Am J Sports Med, 20:507-15.

Frank CB, Jackson DW. 1997. The science of reconstruction of the anterior cruciate ligament. J Bone Joint Surg, 79:1556-76.
Fu FH, Bennett CH, Lattermann C et al. 1999. Current trends in anterior cruciate ligament reconstruction. Part 1: Biology and biomechanics of reconstruction. Am J Sports Med, 27:821-30.

Goulet F, Germain L, Caron C et al. 1997. Tissue-engineered ligament. In Yahia LH, ed. Ligaments and ligamentoplasties. Berlin: SpringerVerlag. p 367-77.

Goulet F, Rancourt D, Cloutier R et al. 1997. Tendons and ligaments. In Lanza R, Langer R, Chick WL, eds. Principles of tissue engineering. 1st ed. San Diego: Academic Pr. p 633-44.

Goulet F, Rancourt D, Cloutier R et al. 2000. Tendons and ligaments. In Lanza R, Langer R, Vacanti J, eds. Principles of tissue engineering. 2nd ed. San Diego: Academic Pr. p 711-22.

Hiemstra LA, Webber S, MacDonald PB et al. 2000. Knee strength deficits after hamstring tendon and patellar anterior cruciate ligament reconstruction. Med Sci Sports Exerc, 32:1472-9.

Hoshi K, Amizuka N, Sakou T et al. 1997. Fibroblasts of spinal ligaments pathologically differentiate into chondrocytes induced by recombinant human bone morphogenetic protein-2: morphological examinations for ossification of spinal ligaments. Bone, 21:155-62.

Huang D, Chang TR, Aggarwal A et al. 1993. Mechanisms and dynamics of mechanical strengthening in ligament-equivalent fibroblastpopulated collagen matrices. Ann Biomed Eng, 21:289-305.

Huynh T, Abraham G, Murray J et al. 1999. Remodeling of an acellular collagen graft into a physiologically responsive neovessel. Nat Biotechnol, 17:1083-6.

Jackson DW, Corsetti J, Simon TM. 1996. Biologic incorporation of allograft anterior cruciate ligament replacements. Clin Orthop, 324:126-33

Jackson DW, Simon TM, Lowery W et al. 1996. Biologic remodeling after anterior cruciate ligament reconstruction using a collagen matrix derived from demineralized bone. An experimental study in the goat model. Am J Sports Med, 24:405-14.

Kanda K, Matsuda T. 1994. Mechanical stress-induced orientation and ultrastructural change of smooth muscle cells cultured in threedimensional collagen lattices. Cell Transplant, 3:481-92.

Kim BS, Nikolovski J, Bonadio J et al. 1999. Cyclic mechanical strain regulates the development of engineered smooth muscle tissue. Nat Biotechnol, 17:979-83.

Kurosaka M, Yoshiya S, Andrish JT. 1987. A biomechanical comparison of different surgical techniques of graft fixation in anterior cruciate ligament reconstruction. Am J Sports Med, 15:225-9.

Langelier E, Rancourt D, Bouchard S et al. 1999. Cyclic traction machine for long-term culture of fibroblast-populated collagen gels. Ann Biomed Eng, 27:67-72.

Nanci A, Zalzal S, Gotoh Y et al. 1996. Ultrastructural characterization and immunolocalization of osteopontin in rat calvarial osteoblast primary cultures. Microsc Res Tech, 33:214-31.

Olson EJ, Kang JD, Fu FH et al. 1988. The biomechanical and histologica effects of artificial ligament wear particles: in vitro and in vivo studies. Am J Sports Med, 16:558-70.

Paquette JS, Goulet F, Boulet LP et al. 1998. Three-dimensional production of bronchi in vitro. Can Respir J, 5:43.

Petite H, Viateau V, Bensaid W et al. 2000. Tissue-engineered bone regeneration. Nat Biotechnol, 18:929-30.

Sabiston P, Frank C, Lam T et al. 1990. Allograft ligament transplantation. A morphological and biochemical evaluation of a medial collateral ligament complex in a rabbit model. Am J Sports Med, 18:160-8.

Woods GA, Indelicato PA, Prevot TJ. 1991. The Gore-Tex anterior cruciate ligament prosthesis. Two versus three year results. Am J Sports Med, 19:48-55.

Yabu M, Takaoka K, Hashimoto J et al. 1992. Immunohistochemical, autoradiographic and electron microscopic studies on the transformation of fibroblasts into chondrocytes in the mouse subfascia induced by bone morphogenetic protein. Histochemistry, 97:463-8. 


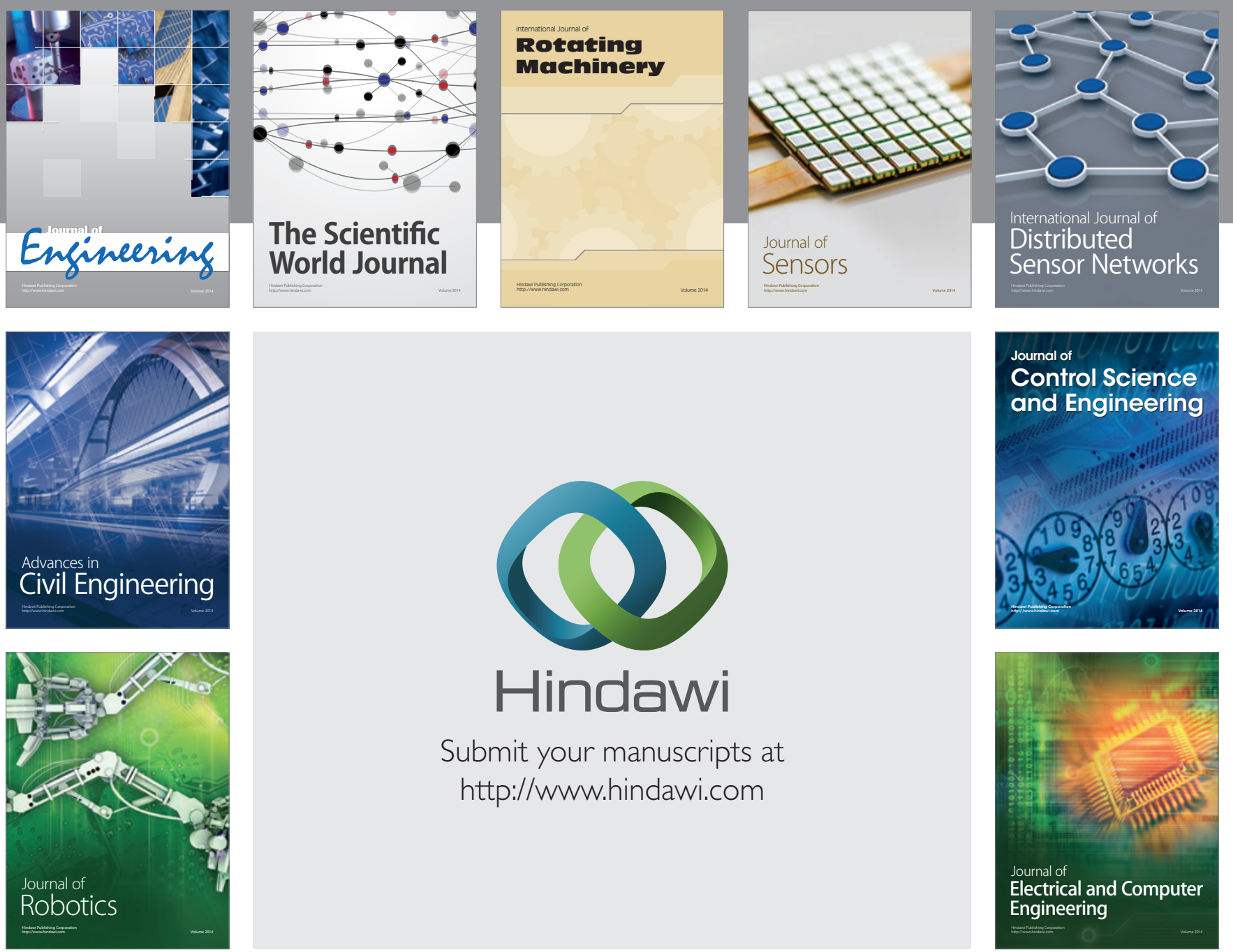

Submit your manuscripts at

http://www.hindawi.com
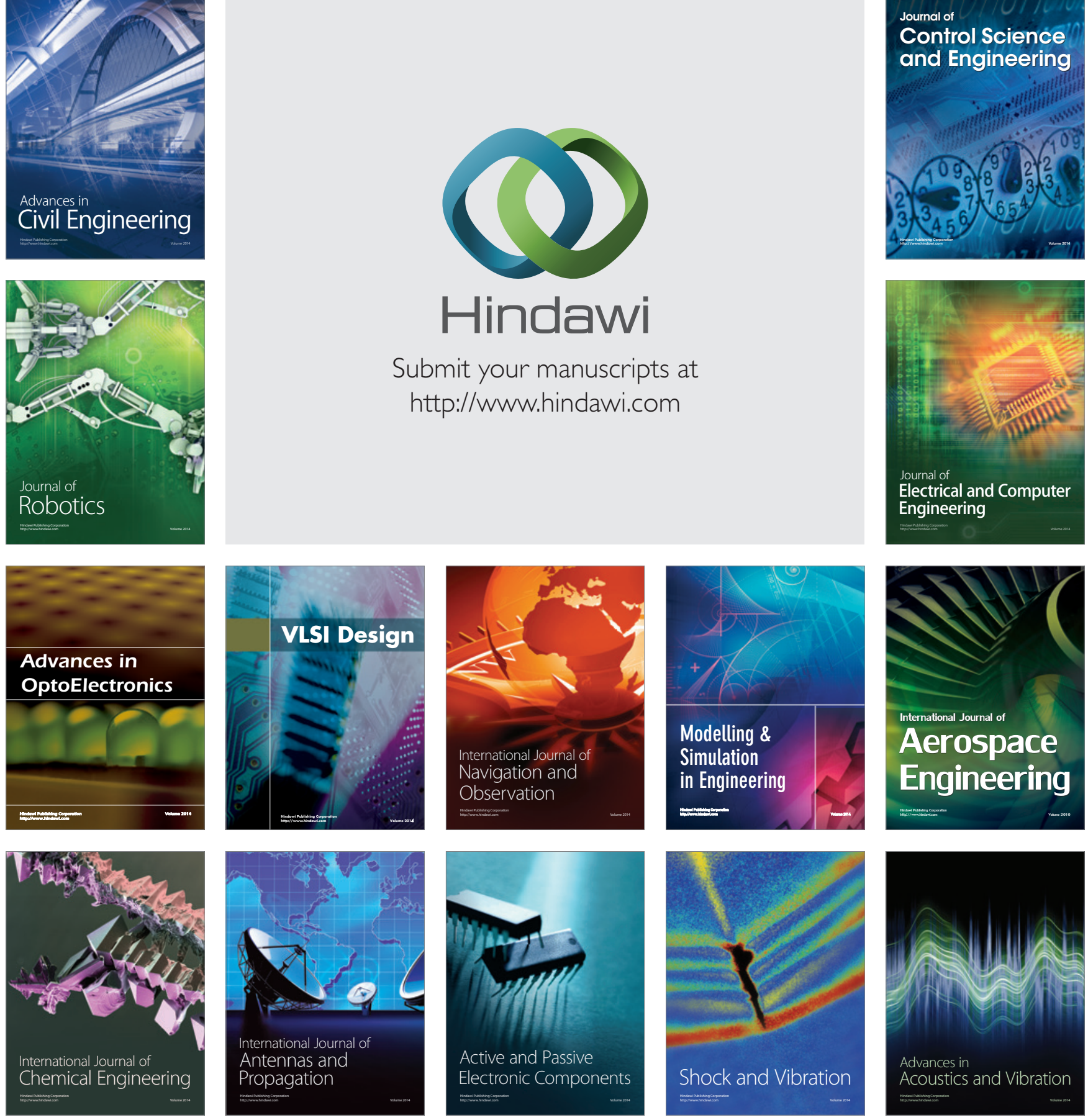